The Corneal Transplant Follow-up Study II (CTFS II): a randomized trial to determine whether HLA class II matching reduces the risk of allograft rejection in penetrating keratoplasty

Armitage WJ, ${ }^{1,2}$ Winton HL, ${ }^{1}$ Jones MNA, ${ }^{2}$ Downward L, ${ }^{2}$ Crewe JM, ${ }^{1}$ Rogers CA, ${ }^{3}$ Tole DM, ${ }^{4}$ Dick $A D^{1,4,5}$

${ }^{1}$ Translational Health Sciences, Bristol Medical School, University of Bristol, Bristol, UK ${ }^{2} \mathrm{NHS}$ Blood \& Transplant, Bristol, UK

${ }^{3}$ Bristol Trials Centre, Bristol Medical School, University of Bristol, Bristol, UK ${ }^{4}$ Bristol Eye Hospital, Bristol, UK

${ }^{5}$ School of Cellular and Molecular Medicine, University of Bristol, Bristol, UK

Corresponding author: $\quad$ Professor W John Armitage

Translational Health Sciences, Bristol Medical School

University of Bristol

Bristol Eye Hospital

Lower Maudlin Street

Bristol BS1 2LX

United Kingdom

\title{
Email: w.j.armitage@bristol.ac.uk
}

Short title: Corneal Transplant Follow-up Study II

Key words: corneal transplantation, allograft rejection, immunological rejection, HLA matching, HLA class II

Word count: 2289

Conflicts of interest: WJA, none; HLW, none; MNAJ, none; LD, none; JMC, none; CAR, none; DMT, none; ADD, consultancies for Novartis, Ally Ltd and Alimera.

Contributorship statement: WJA, Principal investigator with overall responsibility for the study, study design, data analysis and interpretation, drafting and revising paper; HLW, Study Co-ordinator, liaising with participating surgeons, contributed to drafting and review of paper; MNAJ, statistical analysis and data interpretation, contributed to drafting and review of paper; LD, statistical analysis and data interpretation, contributed to drafting and review of paper; JMC, Study Co-ordinator, liaising with participating surgeons, contributed to drafting 
and review of paper; CAR, statistical advice on study design, analysis and data interpretation, contributed to drafting and review of paper; DMT, corneal transplant surgeon, advised on clinical aspects of the study, recruited patients to the study, contributed to the drafting and review of the paper; ADD, Consultant Ophthalmologist and specialist in ocular immunology, advised on clinical and immunological aspects of the study and data interpretation, contributed to drafting and review of paper.

International Standard Randomized Control Trial Number: ISRCTN25094892

National Institute for Health Research (NIHR) Clinical Research Network: UKCRN ID 9871

South West Research Ethics Committee favourable opinion: MREC/97/6/8 National Research Ethics Service: IRAS Project ID 11351

The study was funded principally by the National Eye Research Centre (grant no. SCIAD036) with start-up funding from the NHS Executive South \& West Research and Development Directorate (grant no. R/14/09/06). 


\section{Synopsis}

2 The prospective Corneal Transplant Follow-up Study II (CTFS II) found no influence of HLA 3 class II matching in high-risk penetrating keratoplasty (PK). Younger recipient age markedly 4 increased the risk of allograft rejection.

5 


\section{Abstract}

7 Purpose: A randomized trial to test the hypothesis that HLA class II matching reduces the 8 risk of allograft rejection in high-risk penetrating keratoplasty (PK).

9 Methods: All transplants were matched for HLA class I antigens ( $\leq 2$ mismatches at the A and $\mathrm{B}$ loci) and corneas were allocated to patients by cohort minimization to achieve 0,1 , or 2 HLA class II antigen mismatches. The corneal transplants $(n=1133)$ were followed for 5 years. The primary outcome measure was time to first rejection episode.

Results: Cox regression analysis found no influence of HLA class II mismatching on risk of immunological rejection (HR 1.13; $95 \% \mathrm{Cl} 0.79,1.63 ; \mathrm{p}=0.51)$. The risk of rejection in recipients older than 60 years was halved compared with recipients $\leq 40$ years (HR 0.51 ; $95 \% \mathrm{Cl} 0.36,0.73 ; p=0.0003)$. Rejection was also more likely where cataract surgery had been performed after PK (HR 3.68; 95\% Cl 1.95, 6.93; $\mathrm{p}<0.0001)$. In univariate analyses, pre-operative factors including chronic glaucoma $(p=0.02)$, vascularization $(p=0.01)$, inflammation $(p=0.03)$, ocular surface disease $(p=0.0007)$, and regrafts $(p<0.001)$ all increased the risk of rejection. In the Cox model, however, none of these factors was individually significant but rejection was more likely where $\geq 2$ pre-operative risk factors were present (HR 2.11; 95\% Cl 1.26, 3.47; $\mathrm{p}<0.003)$.

Conclusions: HLA class II matching, against a background of HLA class I matching, did not reduce the risk of allograft rejection. Younger recipient age, the presence of $\geq 2$ pre-operative risk factors and cataract surgery after PK all markedly increased the risk of allograft rejection. 
Allograft rejection remains a serious complication after penetrating keratoplasty (PK), accounting for $30-40 \%$ of graft failures within 1-2 years. ${ }^{1-3}$ Even if treated successfully, just a single rejection episode can jeopardize long-term graft survival. ${ }^{4}$ Topical corticosteroid is the treatment of choice for the prevention and management of corneal transplant rejection; however, it is not always successful at reversing rejection episodes and its long-term use can result in raised intraocular pressure and cataract. ${ }^{5}$ While promising results have been reported with the use of systemic immunosuppressants such as tacrolimus and mycophenolate mofetil in patients at high risk of rejection, ${ }^{6}$ supporting evidence from randomized trials is limited. ${ }^{7}$ Lamellar grafts are less likely than PK to suffer allograft rejection but an underlying risk of rejection, albeit very low for Descemet membrane endothelial keratoplasty (DMEK), remains. ${ }^{89}$ Moreover, there are still many PKs performed worldwide ${ }^{10}$ and alternative approaches for reducing the risk of rejection are needed, ${ }^{11} 12$ which may come through an increased understanding of the immunobiology of corneal transplantation in humans.

Matching for human leucocyte antigens (HLA) between donors and recipients is an effective strategy for reducing the risk of allograft rejection in organ transplantation. ${ }^{13}$ However, the results of studies investigating the influence of HLA matching in corneal transplantation remain equivocal, especially for HLA class II matching where there are reports of a beneficial effect, no effect and even a detrimental effect. ${ }^{14-17}$ This may in part be due to errors in HLA typing using serological methods ${ }^{18}$ since it has been estimated that errors in just $5 \%$ of HLADR tissue types would be sufficient to reduce any benefit of HLA class II matching. ${ }^{14}$ There is also a paucity of large-scale, prospective clinical trials of HLA matching in corneal transplantation and almost all of the available information comes from retrospective analyses of clinical outcome data. Nonetheless, HLA matching is still advocated for corneal transplantation as an approach to reduce the risk of rejection. ${ }^{19} 20$ 
To resolve the uncertainty surrounding the role of HLA class II matching in high risk PK, the corneal transplant follow-up study II (CTFS II) was designed as a randomized clinical trial to determine whether HLA class II matching, against a background of HLA class I matching, reduces the risk of allograft rejection in high-risk PK. ${ }^{21}$ To avoid the inevitable errors with serological methods, DNA-based techniques were used for the tissue typing of all donors and recipients.

MATERIALS AND METHODS

Patients

The design of this randomized clinical trial, the methodology, patient inclusion and exclusion criteria, donor and recipient characteristics, sample size, and allocation to the study groups follow the relevant CONSORT Guidelines (www.consort-statement.org) as described in detail in a previous paper. ${ }^{21}$ The study complied with the tenets of the Declaration of Helsinki. Briefly, patients of either sex aged 16 years or older who met the selection criteria for being at increased risk of PK rejection (regrafts, bullous keratopathy, vascularized cornea, active or past inflammatory/infectious disease, glaucoma) and who had given informed consent to participate in the study, were registered with NHS Blood and Transplant and placed on the waiting list for HLA-matched transplants. The donor corneas were all stored by organ culture at $34^{\circ} \mathrm{C}$ and the minimum endothelial cell density estimated 3 days before surgery was 2200 cells $/ \mathrm{mm}^{2} .{ }^{22}$ All tissue typing of donors and recipients used DNAbased methods (PCR-SSP/SSO). For a given cornea, patients were identified on the waiting list with $\leq 2$ HLA class I (HLA-A and -B combined) antigen mismatches with the donor and then the cornea allocated to one of these patients by cohort minimization, which included a 
Patients were followed for 5 years with data being collected at the time of surgery and postoperatively at 6 months and then on the anniversary of the transplant.

\section{Statistical analysis}

The primary outcome measure was time to first rejection episode, regardless of whether it was treated successfully or resulted directly in graft failure. Pre- and per-operative variables of interest, identified from previous studies, ${ }^{3}$ were examined univariately by comparing Kaplan-Meier curves with the log-rank test. Subsequently, variables were selected for Cox proportional hazards regression analysis by forward selection using the likelihood ratio test, and all variables significant at the $10 \%$ level were included in the final model. The number of HLA class II mismatches and donor sex match were included in the model during variable selection despite lack of significance at the $10 \%$ level because of their relevance to the study. Post-operative factors were modelled as time-dependent variables in the Cox model. Some patients received multiple transplants and recipient was therefore modelled as a random effect to allow for this. Survival estimates and hazard ratios $(\mathrm{HR})$ are quoted with 95\% confidence intervals (95\% Cl).

\section{RESULTS}

Between 3 September 1998 and 2 June 2011, 1133 transplants (all PK) in 980 patients were accrued to the study. Of these 1078 met all the study selection criteria and were randomized to one of the three study groups of $0(n=182), 1(n=483)$ or $2(n=413)$ HLA class II (HLA-DR) mismatches. ${ }^{21}$ Five-year graft survival for these high-risk grafts was $63 \%(95 \% \mathrm{Cl} 60,66)$. The overall rejection-free survival estimate at 5 years was $67 \%(95 \% \mathrm{Cl} 64,71)$. Figure 3 shows the cumulative numbers of events (i.e., first rejection episodes) and numbers of transplants at risk at each follow-up time point. Of the 298 first rejection episodes reported, $79 \%$ occurred within the first 2 years after the transplant, with $12 \%, 6 \%$ and $3 \%$ of first rejection episodes occurring subsequently in post-operative years 3,4 and 5 , respectively. 
107 Univariate analysis

108 Donor factors

109 The only donor factor that influenced risk of rejection was storage time of corneas in organ culture. The Kaplan-Meier rejection free survival estimate at 5 years was higher (i.e., lower risk of rejection) for corneas stored in organ culture $>21$ days $(69 \% ; 95 \% \mathrm{Cl} 64,73)$ than for corneas stored $\leq 15$ days $(55 \% ; 95 \% \mathrm{Cl} 42,67)(\mathrm{p}=0.04)$. Other donor factors, namely age $(p=0.8)$, gender $(p=0.83)$, cornea from male donor into female recipient $(p=0.38)$, donor cause of death $(p=0.6)$, death to enucleation time $(p=0.2)$, and endothelial cell density $(p=0.4)$ had no influence with the risk of rejection.

Pre- and per- operative recipient factors

Recipient age had a marked influence on risk of rejection (Figure 1) with younger patients having a rather lower rejection-free survival at 5 years (i.e., increased risk of rejection) than older patients $(p<0.0001)$. Indication for transplantation $(p<0.0001)$ and preoperative risk factors, including inflammation ( $p=0.03)$, chronic glaucoma $(p=0.02)$, ocular surface disease $(p=0.007)$, deep vascularization $(p=0.01)$, and whether the PK was a regraft $(p<0.0001)$ all increased the risk of rejection (Table 1). Moreover, Figure 2 shows the more risk factors present, the lower the rejection-free survival at 5 years $(p<0.0001)$. Factors not reaching the $10 \%$ level of significance included: number of HLA class I mismatches $(p=0.7)$, total number of HLA class I + HLA class II mismatches $(p=0.8)$, reason for graft to improve vision only $(p=0.26)$, recipient trephine diameter $(p=0.19)$, donor-recipient trephine diameter difference $(p=0.39)$, and cataract extraction $(p=0.22)$ or vitrectomy $(p=0.26)$ at the time of the transplant operation. Finally, the Kaplan-Meier plot in Figure 3 shows that the number of HLA class II mismatches had no influence on rejection-free survival at 5 years $(p=0.6)$. 
Table 1. Kaplan-Meier rejection-free survival estimates at 5 years for significant donor, recipient and transplant factors $(p<0.05)$. Rejection-free survival data for the influence of recipient age, numbers of risk factors, and level of HLA class II mismatch are shown, respectively, in Figures 1-3.

\begin{tabular}{|c|c|c|c|c|}
\hline Factor & $\mathbf{n}$ & Survival (\%) & $95 \% \mathrm{Cl}$ & $\mathbf{p}^{*}$ \\
\hline \multicolumn{4}{|c|}{$\begin{array}{l}\text { DONOR } \\
\text { Corneal storage time in organ culture (days) }\end{array}$} & \multirow[t]{2}{*}{0.04} \\
\hline $\begin{array}{l}<15 d \\
15-21 d \\
22-35 d\end{array}$ & $\begin{array}{r}80 \\
461 \\
521\end{array}$ & $\begin{array}{l}55 \\
67 \\
69\end{array}$ & $\begin{array}{l}42,67 \\
62,71 \\
64,73\end{array}$ & \\
\hline
\end{tabular}

$\begin{aligned} & \text { PRE- AND PER-OPERATIVE } \\ & \text { Inflammation }\end{aligned}$
No
Yes
Co

\section{Chronic glaucoma}

No

Yes

Ocular surface disease

No

Yes

Deep vascularization

No

Yes

Regraft ${ }^{*}$

No

Yes

Indication (original indication if regraft) $\$$

Ectasias

Dystrophies

Previous ocular surgery

Infection

Injury

Opacification

Other

450

627

497

580

74

227

310

197

75

61

133

$\begin{array}{lll}816 & 69 & 65,73 \\ 261 & 60 & 53,67\end{array}$

0.03

$908 \quad 68 \quad 65,72$

$\begin{array}{lll}169 & 59 & 50,67\end{array}$

70

63

65,73

57,68

73

62

68,77

57,66

60

72

69

69

40

69

67
0.02

0.007

$<0.0001$

$<0.0001$

${ }^{\star}$ Comparing rejection-free survival up to 5 years after transplantation.

¥Ipsilateral regraft was considered a risk factor rather than a specific indication for transplantation. Regrafts were therefore included in the list of indications under their original indication for transplantation.

$\S^{\S}$ Ectasias (70 keratoconus, 2 keratoglobus, 2 'other'); Dystrophies (166 Fuchs endothelial dystrophy, 61 'other'); Previous ocular surgery (227 pseudophakic bullous keratopathy, 53 aphakic corneal oedema, 30 'other'). 


\section{Cox proportional hazards regression}

129 The final Cox model is shown in Table 2. For recipients aged 40 years or younger, the risk of 130 rejection was approximately double that for patients aged between 61 and 80 years (HR $1310.51 ; 95 \% \mathrm{Cl} 0.36,0.73 ; \mathrm{p}=0.0003)$ and for patients over 80 years old (HR $0.49 ; 95 \% \mathrm{Cl}$ $1320.30,0.81 ; p=0.005)$. The presence of 2 or more preoperative risk factors more than doubled 133 the risk of rejection (HR $2.11 ; 95 \% \mathrm{Cl} 1.28,3.47 ; p=0.003)$. Cataract surgery after the 134 corneal transplant was associated with a more than threefold increased risk of rejection (HR $1353.68 ; 95 \% \mathrm{Cl} 1.95,6.93 ; \mathrm{p}<0.0001)$ : it was not known whether patients were on topical 136 steroid at the time of cataract surgery. When controlling for other factors in the final Cox 137 model, HLA class II matching had no influence on risk of rejection (e.g., for 2 vs 0 138 mismatches: HR 1.13; 95\% Cl 0.79, 1.63; $p=0.51$ ) (Table 2 and Figure 3). 
Table 2. Final Cox model for risk of allograft rejection over a 5-year period adjusting for recipient as a random effect to account for patients with multiple grafts ${ }^{*}(n=1078)$.

\begin{tabular}{|c|c|c|c|c|}
\hline Factor $§$ & $\mathbf{n}$ & HR & $95 \% \mathrm{Cl}$ & $\mathbf{p}$ \\
\hline \multicolumn{5}{|c|}{ HLA class II mismatches $(p=0.4)$} \\
\hline 0 & 182 & 1.00 & - & - \\
\hline 1 & 483 & 1.02 & $0.71,1.47$ & 0.91 \\
\hline 2 & 413 & 1.13 & $0.79,1.63$ & 0.51 \\
\hline \multicolumn{5}{|c|}{ Number of pre-operative risk factors $(p=0.0005)$} \\
\hline 0 & 136 & 1.00 & - & - \\
\hline 1 & 293 & 1.49 & $0.89,2.50$ & 0.13 \\
\hline 2 & 347 & 2.11 & $1.28,3.47$ & 0.003 \\
\hline $3+$ & 301 & 2.79 & $1.69,4.60$ & $<0.0001$ \\
\hline \multicolumn{5}{|l|}{ Recipient age $(p=0.0015)$} \\
\hline$\leq 40$ & 161 & 1.00 & - & - \\
\hline $41-60$ & 295 & 0.71 & $0.49,1.02$ & 0.064 \\
\hline $61-80$ & 480 & 0.51 & $0.36,0.73$ & 0.0003 \\
\hline$\geq 81$ & 141 & 0.49 & $0.30,0.81$ & 0.0053 \\
\hline \multicolumn{5}{|l|}{ Donor-recipient sex match } \\
\hline All other matches & 801 & 1.00 & - & - \\
\hline Male donor to female recipient & 276 & 0.96 & $0.72,1.30$ & 0.81 \\
\hline \multicolumn{5}{|c|}{ Selective adjustment/removal of sutures ${ }^{*}$} \\
\hline No & 562 & 1.00 & - & - \\
\hline Yes & 516 & 0.77 & $0.56,1.06$ & 0.11 \\
\hline \multicolumn{5}{|c|}{ Cataract surgery after transplant } \\
\hline No & 1005 & 1.00 & - & - \\
\hline Yes & 73 & 3.68 & $1.95,6.93$ & $<0.0001$ \\
\hline
\end{tabular}

Random effect for transplant recipient accounting for multiple grafts in the same recipient $(p=0.06)$

*Multiple grafts include both ipsilateral and contralateral grafts in the same recipient.

§Factors considered but not included in the final Cox model as $p>0.1$ :

Donor factors: donor age $(p=0.13)$, corneal storage time $(p=0.18)$, endothelial cell density $(p=0.21)$

Pre- and per-operative factors: indication $(p=0.19)$, deep vascularization $(p=0.65)$, regraft $(p=0.24)$, inflammation $(p=0.53)$, glaucoma $(p>0.99)$, ocular surface disease $(p=0.53)$, recipient trephine diameter $(p=0.16)$, donor-recipient trephine difference $(p=0.80)$, suturing method $(p=0.71)$

Postoperative factors: wound leak $(p=0.44)$, glaucoma medication $(p=0.65)$, other immunosuppresants $(p=0.18)$, elective removal of all sutures $(0.53)$, loose or broken stitch $(p=0.12)$

${ }^{¥}$ Removal of interrupted or a continuous suture where a double-running suture technique was used, or adjustment of a continuous suture to even out tension. 
We found no influence of HLA class II matching on risk of rejection in this randomized study of a large cohort of high-risk, full-thickness corneal transplants. Previous studies that observed a benefit or detrimental effect of class II matching are therefore not supported by the present findings. In marked contrast to renal transplantation, ${ }^{13}$ the lack of influence of HLA class II matching suggests that the direct pathway of allorecognition is not activated in corneal transplantation, ${ }^{23}$ perhaps owing in part to a lack of professional antigen presenting cells (APC) in the corneal graft. The cornea is not devoid of APCs: Langerhans cells are present in the corneal epithelium but are confined to the periphery of normal corneas and therefore not transplanted in significant numbers in a corneal graft. Despite this, storage time of donor corneas in organ culture has been postulated to reduce corneal graft immunogenicity through loss of APCs during prolonged storage. In the univariate analyses, longer storage time in organ culture was indeed associated with reduced risk of rejection $(p=0.04)$, but this was not the case in the Cox model when other factors were controlled for. The presence of immature dendritic cells (DC) in the central cornea has been reported in mice. ${ }^{24} 25$ Under certain conditions, such as transplantation of a cornea into an inflamed graft bed, these cells mature to express HLA class II antigens and activate host T-cells by the direct pathway. In the present study, the lack of influence of HLA class II matching would suggest that either a similarly immature population of DCs is not present in human cornea or there was a failure to stimulate maturation even though CTFS II included only high-risk grafts.

While the potential benefit or otherwise of HLA class I matching could not be evaluated in our study, further studies in mice have suggested little influence of major histocompatibility (MHC) mismatches in corneal transplant rejection, despite evidence to the contrary from some human studies. ${ }^{14}$ Instead, non-MHC antigen mismatches have been shown to play a major role in rejection in mice. ${ }^{26}$ The role of non-MHC mismatches is more difficult to 
elucidate in humans. Nonetheless, reports of increased risk of rejection when corneas from male donors, expressing the HLA class I restricted $\mathrm{H}-\mathrm{Y}$ antigen, are transplanted into female recipients suggest that non-MHC antigens may have a role in stimulating allograft rejection of human corneal transplants. ${ }^{27}$ However, inclusion of donor-recipient sex match in our CTFS II Cox model failed to show an increased risk of rejection when female recipients received corneas from male donors $(p>0.99)$. Other factors such as cytokine gene polymorphisms may influence transplant outcome. Tumour necrosis alpha (TNF- $\alpha$ ) and interleukin-10 (IL-10) polymorphisms have been shown to be associated with transplantrelated death in stem cell transplantation. ${ }^{28}$ Using a subset of CTFS II recipients we found two TNF-a haplotypes one of which was associated with increased and the other with decreased risk of rejection in these high-risk corneal graft recipients. ${ }^{29}$

The use of systemic immunosuppressants in addition to topical corticosteroids was not significant in the Cox model $(p=0.18)$ (Table 2). A Cochrane Review highlighted the lack of strong evidence for systemic immunosuppression in corneal transplantation, ${ }^{7}$ although there are reports of its value in high-risk cases. ${ }^{6}$

The presence of blood vessels in the cornea before transplantation has typically been considered to be a risk factor for rejection in $\mathrm{PK}^{2}$ However, even though we found vascularization in the cornea before $\mathrm{PK}$ to increase the risk of rejection in the univariate analyses, this observation was not confirmed in the Cox model. Since the detection of lymph vessels in the cornea, ${ }^{30}$ the relative importance of haem- vs. lymphangiogensis in corneal transplant rejection has been discussed and may help to explain, at least in part, our failure to see a clear influence of vascularization in our study of high risk PK. ${ }^{31} 32$ Individual preoperative risk factors were not significant in the multivariate Cox regression analysis but the risk of rejection did increase when two or more of these risk factors were present (Fig. 2 
and Table 2). The most frequent combinations of risk factors were regraft and vascularization ( $n=197)$, vascularization, glaucoma and regraft $(n=78)$, and vascularization and infection $(n=76)$.

We found a marked reduction in risk of rejection with increasing recipient age (Figure 1 and Table 2), which supports an earlier retrospective study in the UK that reported a similar finding. ${ }^{3}$ It is well-established that both the innate and acquired immune systems change with age, a process termed immunosenescence. As a result, the elderly have an increased susceptibility to infection and inflammatory disease, and poorer response to vaccination, which is where most of the research into this phenomenon has been directed. ${ }^{33}$ In particular, there are alterations in DC subsets, a reduction in peripheral naïve T-cells, an increase in memory T-cells and alterations in cytokine expression, which overall leads to a reduced ability to respond adequately to novel antigens. There have been some studies of the effects of immunosenescence in transplantation, which show reduced risk of acute rejection in older kidney recipients. ${ }^{34} 35$ These changes to the immune system with age may help to explain our findings of reduced risk of rejection in recipients over 60 years old.

In summary, unlike in renal transplantation, HLA class II matching did not reduce the risk of rejection in high-risk, full-thickness corneal transplantation. Other factors are therefore likely to play an important role in corneal transplant immunology and a project (VISICORT) is currently underway to find adverse immune signatures in corneal transplant patients. ${ }^{36}$ The reduced risk of rejection with increasing recipient age certainly warrants further investigation.

\section{ACKNOWLEDGEMENTS}


216 We thank all the contributing surgeons (listed in reference 21) and their patients who gave

217 their consent to participate in this study. We also thank Rachel Johnson, Elinor Curnow and

218 Cathy Hopkinson, NHS Blood and Transplant, for their support and further statistical advice.

219 


\section{REFERENCES}

1. Williams KA, Coster DJ. The immunobiology of corneal transplantation. Transplantation 2007;84:806-13.

2. Williams KA, Keane MC, Coffey NE, et al. The Australian Corneal Graft Registry Report 2018, http://hdl.handle.net/2328/37917 (accessed 6 Jul 2020).

3. Vail A, Gore SM, Bradley BA, et al. Conclusions of the corneal transplant follow up study. Brit J Ophthalmol 1997;81:631-6.

4. Coster DJ, Williams KA. The impact of corneal allograft rejection on the long-term outcome of corneal transplantation. Am J Ophthalmol 2005;140:1112-22.

5. Kharod-Dholakia B, Randleman JB, Bromley JG, et al. Prevention and treatment of corneal graft rejection: current practice patterns of the Cornea Society (2011). Cornea 2015;34:609-14.

6. Chow SP, Cook SD, Tole DM. Long-term outcomes of high-risk keratoplasty in patients rReceiving systemic immunosuppression. Cornea 2015;34:1395-9.

7. Abudou M, Wu T, Evans JR, et al. Immunosuppressants for the prophylaxis of corneal graft rejection after penetrating keratoplasty. Cochrane Database Syst Rev 2015(8):CD007603.

8. Anshu A, Price MO, Price FW, Jr. Risk of corneal transplant rejection significantly reduced with Descemet's membrane endothelial keratoplasty. Ophthalmology 2012;119:53640.

9. Jones MN, Armitage WJ, Ayliffe W, et al. Penetrating and deep anterior lamellar keratoplasty for keratoconus: a comparison of graft outcomes in the United kingdom. Invest Ophth Vis Sci 2009;50:5625-9. 
10. Gain P, Jullienne R, He Z, et al. Global Survey of Corneal Transplantation and Eye Banking. JAMA Ophthalmol 2016;134:167-73.

11. Coster DJ, Jessup CF, Williams KA. Mechanisms of corneal allograft rejection and regional immunosuppression. Eye (Lond) 2009;23:1894-7.

12. Yu T, Rajendran V, Griffith M, et al. High-risk corneal allografts: A therapeutic challenge. World J Transplant 2016;6:10-27.

13. Opelz G, Döhler B. Effect of human leukocyte antigen compatibility on kidney graft survival: comparative analysis of two decades. Transplantation 2007;84:137-43.

14. Völker-Dieben HJ, Claas FH, Schreuder GM, et al. Beneficial effect of HLA-DR matching on the survival of corneal allografts. Transplantation 2000;70:640-8.

15. The Collaborative Corneal Transplantation Studies (CCTS). Effectiveness of histocompatibility matching in high-risk corneal transplantation. The Collaborative Corneal Transplantation Studies Research Group. Arch Ophthalmol 1992;110:1392403.

16. Gore SM, Vail A, Bradley BA, et al. HLA-DR matching in corneal transplantation. Systematic review of. Transplantation 1995;60:1033-9.

17. Bradley BA, Vail A, Gore SM, et al. Negative effect of HLA-DR matching on corneal transplant rejection. Transplant Proc 1995;27:1392-4.

18. Hopkins KA, Maguire MG, Fink NE, et al. Reproducibility of HLA-A, B, and DR typing using peripheral blood samples: results of retyping in the Collaborative Corneal Transplantation Studies. Human Immunology 1992;33:122-8.

19. Vitova A, Kuffova L, Klaska IP, et al. The high-risk corneal regraft model: a justification for tissue matching in humans. Transpl Int 2013;26:453-61. 
20. van Essen TH, Roelen DL, Williams KA, et al. Matching for Human Leukocyte Antigens (HLA) in corneal transplantation - to do or not to do. Prog Retin Eye Res 2015;46:84110.

21. Armitage WJ, Winton HL, Jones MNA, et al. Corneal transplant follow-up study II (CTFS II): a prospective clinical trial to determine the influence of HLA class II matching on corneal transplant rejection: baseline donor and recipient characteristics. Brit $J$ Ophthalmol 2019;103:132-36.

22. Armitage WJ, Jones MN, Zambrano I, et al. The suitability of corneas stored by organ culture for penetrating keratoplasty and influence of donor and recipient factors on 5year graft survival. Invest Ophth Vis Sci 2014;55:784-91.

23. Batchelor JR. The laws of transplantation: a modern perspective. Eye 1995;9:152-4.

24. Hamrah P, Dana MR. Corneal antigen-presenting cells. Chem Immunol Allergy 2007;92:58-70.

25. Hamrah $P$, Liu $Y$, Zhang Q, et al. The corneal stroma is endowed with a significant number of resident dendritic cells. Invest Ophth Vis Sci 2003;44:581-9.

26. Nicholls SM, Williams NA. MHC matching and mechanisms of alloactivation in corneal transplantation. Transplantation 2001;72:1491-7.

27. Böhringer D, Spierings E, Enczmann J, et al. Matching of the minor histocompatibility antigen $\mathrm{HLA}-\mathrm{A} 1 / \mathrm{H}-\mathrm{Y}$ may improve prognosis in corneal transplantation. Transplantation 2006;82:1037-41.

28. Keen LJ, DeFor TE, Bidwell JL, et al. Interleukin-10 and tumor necrosis factor alpha region haplotypes predict transplant-related mortality after unrelated donor stem cell transplantation. Blood 2004;103:3599-602. 
29. Winton HL, Bidwell JL, Armitage WJ. Functional tumor necrosis factor alpha polymorphisms and haplotype analysis in high-risk corneal transplantation. Transplant Proc 2014;46:1548-53.

30. Cursiefen C, Schlötzer-Schrehardt U, Küchle M, et al. Lymphatic vessels in vascularized human corneas: immunohistochemical investigation using LYVE-1 and podoplanin. Invest Ophth Vis Sci 2002;43:2127-35.

31. Zhong W, Montana M, Santosa SM, et al. Angiogenesis and lymphangiogenesis in corneal transplantation-A review. Surv Ophthalmol 2018;63:453-79.

32. Clahsen T, Buttner C, Hatami N, et al. Role of Endogenous Regulators of Hem- And Lymphangiogenesis in Corneal Transplantation. J Clin Med 2020;9:479-99.

33. Aiello A, Farzaneh F, Candore G, et al. Immunosenescence and Its Hallmarks: How to Oppose Aging Strategically? A Review of Potential Options for Therapeutic Intervention. Front Immunol 2019;10:2247

34. Colvin MM, Smith CA, Tullius SG, et al. Aging and the immune response to organ transplantation. J Clin Invest 2017;127:2523-29.

35. Martins PN, Tullius SG, Markmann JF. Immunosenescence and immune response in organ transplantation. Int Rev Immunol 2014;33:162-73.

36. Armitage WJ, Goodchild C, Griffin MD, et al. High-risk corneal cransplantation: recent developments and future possibilities. Transplantation 2019;103:2468-78. 


\section{FIGURE LEGENDS}

Figure 1. Kaplan-Meier plot for rejection-free survival stratified by recipient age and 5-year rejection-free survival estimates.

Figure 2. Kaplan-Meier plot for rejection-free survival stratified by number of pre-operative risk factors (regraft, vascularization, glaucoma, inflammation, ocular surface disease and 'other') and 5-year rejection-free survival estimates.

Figure 3. Kaplan-Meier plot for rejection-free survival stratified by number of HLA class II mismatches, 5-year rejection-free survival estimates, numbers of events (i.e., first rejection episode) and numbers of transplants at risk at each postoperative follow-up time. 


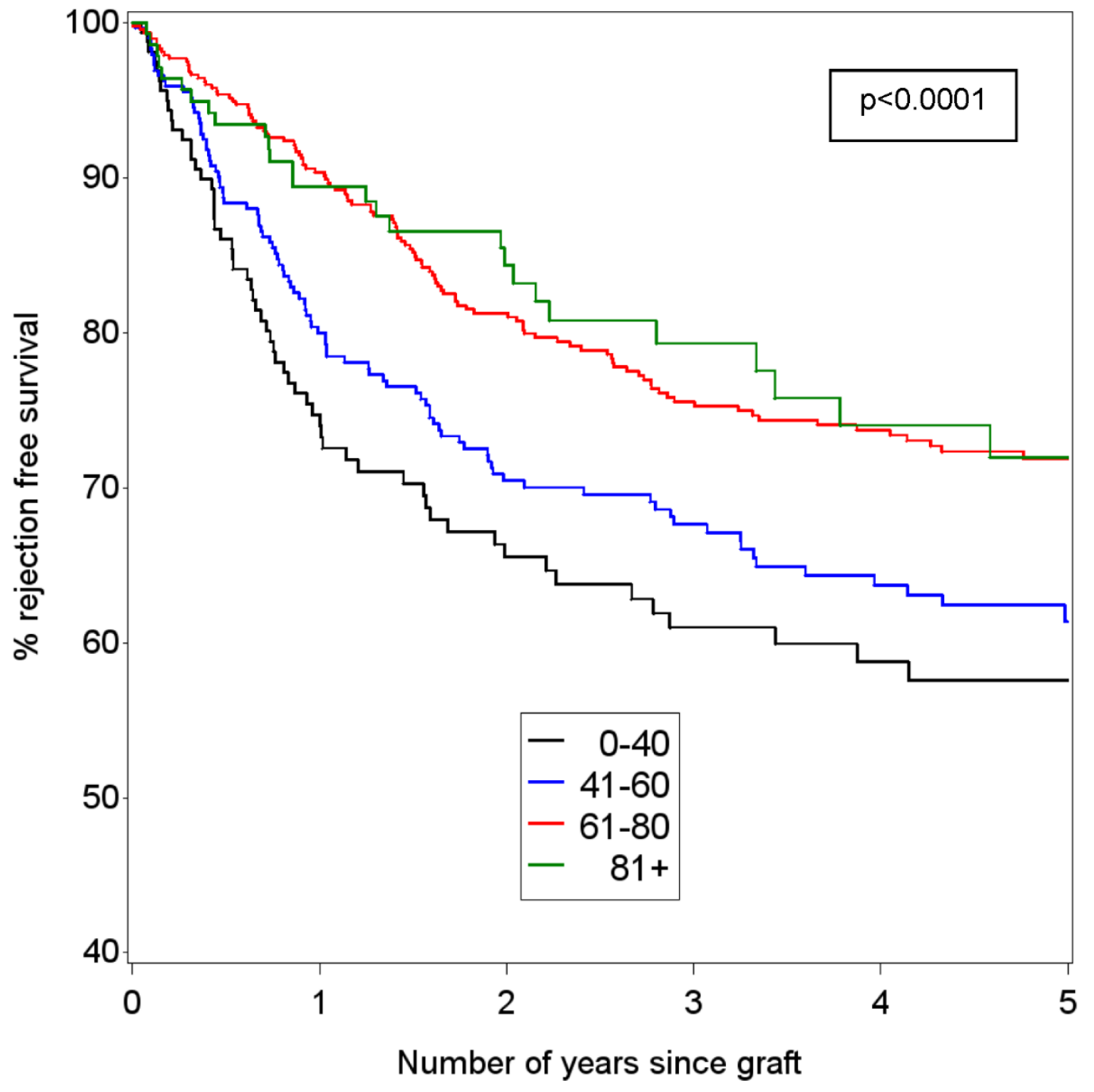

\begin{tabular}{|c|c|c|c|}
\hline \multirow[t]{2}{*}{ Recipient age } & \multirow[t]{2}{*}{$\mathbf{n}$} & \multicolumn{2}{|c|}{$\begin{array}{c}\text { 5-year rejection-free survival } \\
(\%)\end{array}$} \\
\hline & & Estimate & $95 \% \mathrm{Cl}$ \\
\hline $0-40$ years & 161 & 58 & 49,66 \\
\hline $41-60$ years & 295 & 61 & 55,67 \\
\hline $61-80$ years & 480 & 72 & 67,76 \\
\hline $81+$ years & 141 & 72 & 61,81 \\
\hline
\end{tabular}

Figure 1. Kaplan-Meier plot for rejection-free survival stratified by recipient age and 5-year rejection-free survival estimates. 


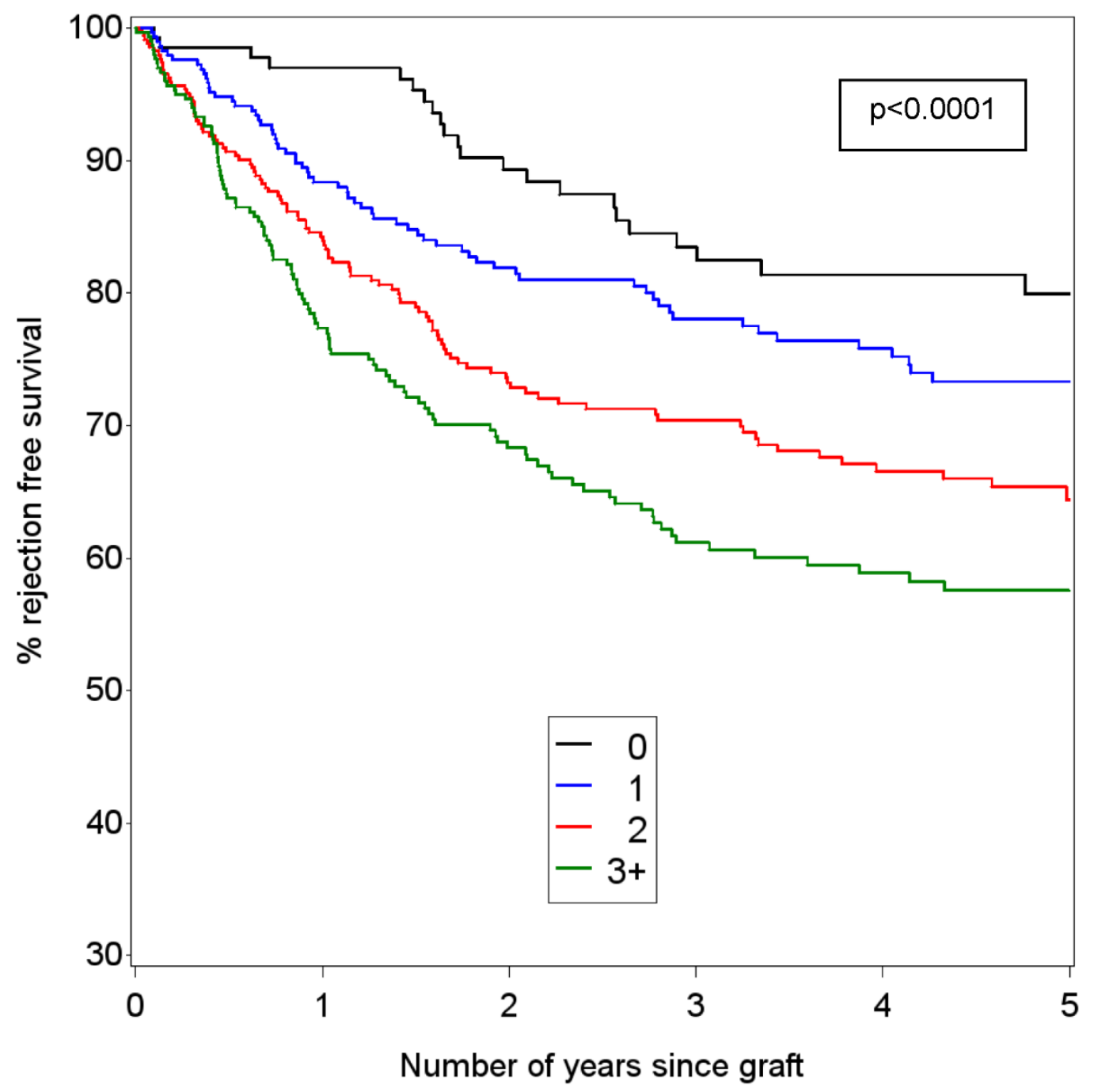

\begin{tabular}{|l|c|c|c|}
\hline Number of risk factors & $\mathbf{n}$ & \multicolumn{2}{|c|}{$\begin{array}{c}\text { 5-year rejection-free } \\
\text { survival (\%) }\end{array}$} \\
\cline { 3 - 4 } & & Estimate & $95 \% \mathrm{Cl}$ \\
\hline 0 & 136 & 80 & 71,86 \\
\hline 1 & 293 & 73 & 67,79 \\
\hline 2 & 347 & 64 & 58,70 \\
\hline $3+$ & 301 & 58 & 51,64 \\
\hline
\end{tabular}

Figure 2. Kaplan-Meier plot for rejection-free survival stratified by number of pre-operative risk factors (regraft, vascularization, glaucoma, inflammation, ocular surface disease and 'other') and 5-year rejection-free survival estimates. 


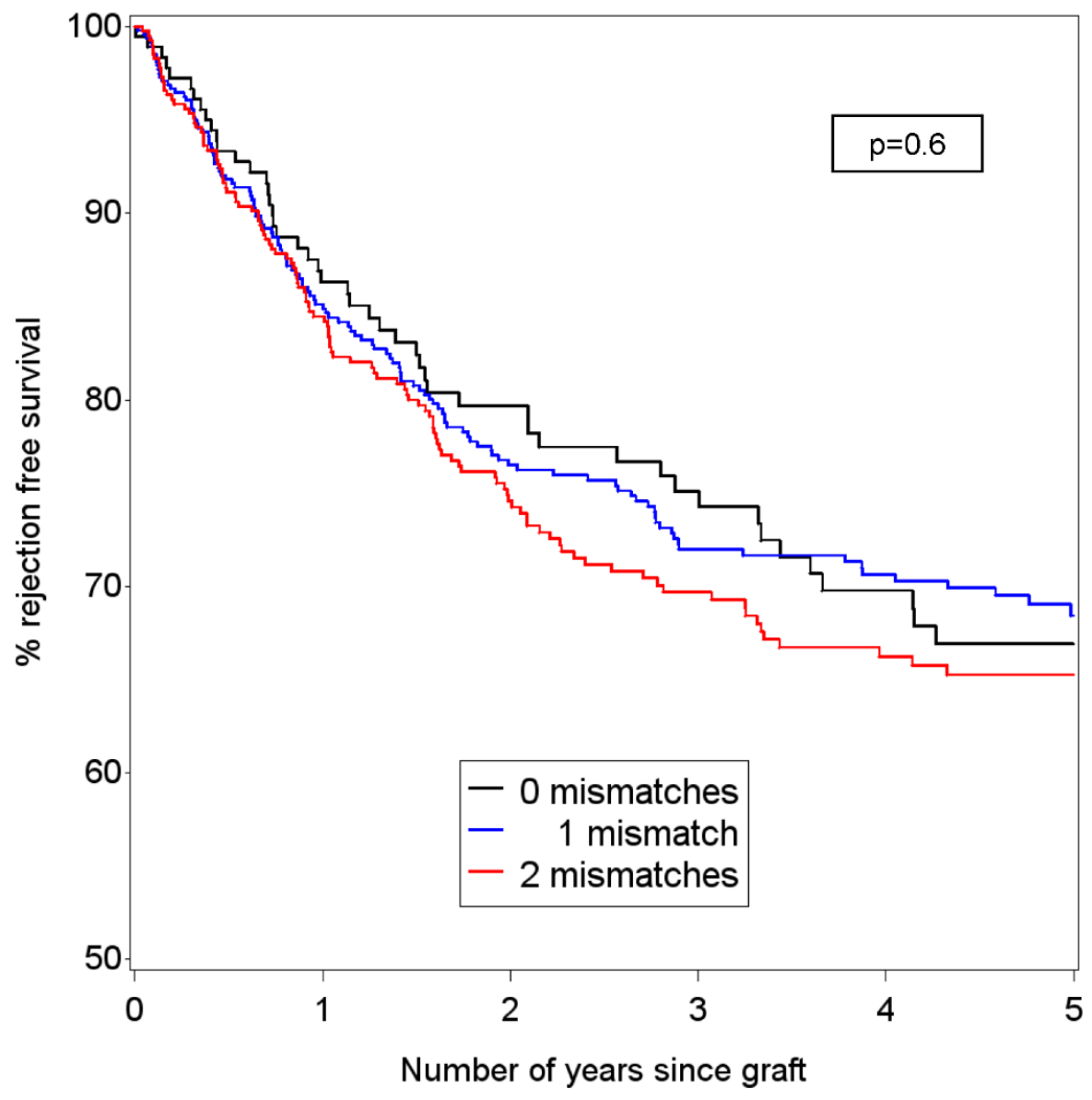

\begin{tabular}{|c|c|c|c|}
\hline \multirow[t]{2}{*}{$\begin{array}{l}\text { HLA Class II match } \\
\text { grade }\end{array}$} & \multirow[t]{2}{*}{$n$} & \multicolumn{2}{|c|}{$\begin{array}{c}\text { 5-year rejection-free } \\
\text { survival }(\%)\end{array}$} \\
\hline & & Estimate & $95 \% \mathrm{C}$ \\
\hline 0 mismatches & 182 & 67 & $58-74$ \\
\hline 1 mismatch & 482 & 68 & $63-73$ \\
\hline 2 mismatches & 413 & 65 & $60-70$ \\
\hline
\end{tabular}

\begin{tabular}{|c|c|c|c|c|c|c|c|}
\hline $\begin{array}{l}\text { Number of years } \\
\text { since graft }\end{array}$ & 0 & 0.5 & 1 & 2 & 3 & 4 & 5 \\
\hline \multicolumn{8}{|l|}{$\begin{array}{l}\text { HLA class II match } \\
\text { grade }\end{array}$} \\
\hline \multicolumn{8}{|l|}{0 mismatches } \\
\hline Number at risk & 182 & 164 & 142 & 110 & 89 & 73 & 33 \\
\hline Cumulative events & 0 & 12 & 24 & 34 & 40 & 46 & 49 \\
\hline \multicolumn{8}{|l|}{1 mismatch } \\
\hline Number at risk & 482 & 429 & 364 & 291 & 240 & 198 & 101 \\
\hline Cumulative events & 0 & 39 & 69 & 104 & 120 & 124 & 129 \\
\hline \multicolumn{8}{|l|}{2 mismatches } \\
\hline Number at risk & 413 & 363 & 314 & 228 & 174 & 138 & 65 \\
\hline Cumulative events & 0 & 36 & 62 & 96 & 110 & 118 & 120 \\
\hline
\end{tabular}


Figure 3. Kaplan-Meier plot for rejection-free survival stratified by number of HLA class II mismatches, 5-year rejection-free survival estimates, numbers of events (i.e., first rejection episode) and numbers of transplants at risk at each postoperative follow-up time. 